\title{
Insufficient Lymph Drainage Causes Abnormal Lipid Accumulation and Vein Wall Degeneration
}

\author{
Hiroki Tanaka, MD, PhD, 1,2,3 Naoto Yamamoto, MD, PhD, ${ }^{1}$ Minoru Suzuki, MD, PhD, ${ }^{1}$ \\ Yuuki Mano, MD, PhD, ${ }^{1}$ Masaki Sano, MD, PhD, ${ }^{1}$ Nobuhiro Zaima, PhD, ${ }^{2,4}$ \\ Takeshi Sasaki, PhD, ${ }^{5}$ Mitsutoshi Setou, MD, PhD, ${ }^{2}$ and Naoki Unno, MD, PhD ${ }^{1}$
}

Objective: Previously, we analyzed human varicose veins (VV) using imaging mass spectrometry (IMS) and detected the abnormal accumulation of lipid molecules in the walls of $\mathrm{V}$, possibly due to insufficient lipid drainage by the lymphatic vessels. In this study, we created an animal model of lymphatic insufficiency to investigate the effects of insufficient lymph drainage on vein walls.

Methods: In rats, the lymphatic collecting vessels surrounding the femoral vein were ligated on one side (the model tissue), which caused the local retention of lymphatic fluid in the perivascular tissue. The equivalent contralateral tissue was used as a control. A histological study of the femoral vein and the surrounding perivascular tissue was conducted. IMS was used to analyze the distribution of lipid molecules in the perivascular tissue.

Results: Fourteen days after the procedure, the lymphatic vessels in the model tissue were significantly dilated. Furthermore, IMS revealed that the composition of the lipid molecules in the perivascular regions of the model tissue had altered. Compared with the control tissue, the model tissue exhibited marked perivascular accumulation of lysophosphatidylcholine (1-acyl 16:0), phosphatidylcholine (16:0/20:4), and triglycerides (52:2). Interestingly, the walls of the femoral veins running through the model tissue were 3.4-fold thicker than those of the femoral veins running through the control tissue. The number of tumor necrosis factor $\alpha$-positive adipocytes was increased in the perivascular regions of the model tissue.

${ }^{1}$ Division of Vascular Surgery, Hamamatsu University School of Medicine, Hamamatsu, Shizuoka, Japan

${ }^{2}$ Department of Cellular \& Molecular Anatomy, Hamamatsu University School of Medicine, Hamamatsu, Shizuoka, Japan ${ }^{3}$ Department of Medical Physiology, Hamamatsu University School of Medicine, Hamamatsu, Shizuoka, Japan

${ }^{4}$ Department of Applied Biological Chemistry, Graduate School of Agricultural Science, Kinki University, Osaka, Japan

${ }^{5}$ Department of Organ \& Tissue Anatomy, Hamamatsu

University School of Medicine, Hamamatsu, Japan

Received: November 10, 2016; Accepted: November 11, 2016 Corresponding author: Naoki Unno, MD, PhD. Division of Vascular Surgery, Hamamatsu University School of Medicine 1-20-1 Handayama, Higashi-ku, Hamamatsu 431-3192, Japan Tel: +81-53-435-2279, Fax: +81-53-435-2273

E-mail: unno@hama-med.ac.jp

This is a translation of Jpn J Phlebol 2015; 26: 227-235.
Conclusion: The findings of this study indicated that the accumulation of lymphatic fluid due to insufficient lymph drainage changes the structure of vein walls, and such changes might be associated with chronic venous insufficiency. (This is a translation of Jpn J Phlebol 2015; 26: 227-235.)

Keywords: varicose veins, lymphedema, phlebolymphology, indocyanine green fluorescence imaging

\section{Introduction}

The pathogenesis of chronic venous insufficiency-related lymphatic dysfunction remains unknown. ${ }^{1,2)}$ We should develop a deeper understanding of the concept of "phlebolymphology." Phlebolymphedema is a condition involving a mixture of venous and lymphatic insufficiency. The presence of lymphatic insufficiency gradually impairs the function of the venous system. ${ }^{3)}$ However, we demonstrated that impaired lymphatic function recovered after the great saphenous vein was stripped in patients with varicose veins (VV). ${ }^{4)}$ Therefore, venodynamics and lymphodynamics might interact to form an inseparable and mutually dependent dual-outflow system in vivo. However, the mechanisms underlying such interactions are complex. Since our knowledge regarding the pathophysiology of venous/lymphatic disorders is solely based on clinical studies, ${ }^{4-6)}$ there has never been any basic research into the changes that occur in the venous and lymphatic systems over time. Therefore, the mechanisms responsible for "phlebolymphological" interactions at the cellular or molecular level are poorly understood. In this study, we recreated an animal model of lymphatic insufficiency to investigate the effects of insufficient lymph drainage on vein walls.

\section{Materials and Methods}

\section{Creation of lymphatic fluid stasis and sample collection}

The animal care procedures and experiments were performed in accordance with the guidelines of the 
Hamamatsu University School of Medicine animal care committee at the Center for Animal Care. Male SpragueDawley rats (8-week old) were purchased from SLC (Shizuoka, Japan) and provided with food and water. The rats were anesthetized using diethyl ether and pentobarbital. In brief, $0.2 \mathrm{ml}$ of Evans blue dye was subcutaneously injected into the rats' dorsalis pedis arteries (Fig. 1). Then, a few lymphatic vessels around the femoral arteries and veins were identified based on the flow pattern of the Evans blue dye. The analyzed region was located in the center of the femoral area, and included the femoral artery, femoral vein, and a few lymphatic vessels. The lymphatic vessels were ligated by placing 8-0 Prolene sutures at $10 \mathrm{~mm}$ proximal and distal from the target region, which was not isolated from the perivascular tissue (Fig. 1). The perivascular tissue surrounding the ligated lymphatic vessels was used to assess the leakage and accumulation of lymphatic fluid and that on the contralateral side was used as the control tissue. Both sets of perivascular tissue were harvested at 7,14 , or 28 days after the procedure. The tissues were frozen in isopentane and stored at $-80^{\circ} \mathrm{C}$. This method enabled an assessment of the distribution of lipid molecules in the tissues to be performed using matrix-assisted laser desorption/ ionization-imaging mass spectrometry (MALDI-IMS).

\section{Immunohistochemistry}

The perivascular tissues were harvested and cut with a cryostat (CM1950; Leica Microsystems GmbH, Wetzlar, Germany) into 8- $\mu \mathrm{m}$ thick sections. The sections were then fixed with $4 \%$ paraformaldehyde in phosphate-buffered saline (PBS; $\mathrm{pH}: 7.4$ ) for $10 \mathrm{~min}$ at room temperature. For conventional immunostaining, slides containing the sections were incubated at room temperature with the following primary antibodies: rabbit anti-Smith-McCort dysplasia (SMC) (1:50, Thermo Fisher Scientific, Waltham, MA, USA), mouse anti-podoplanin (1:100; ReliaTech $\mathrm{GmbH}$, Braunschweig, Germany), rabbit anti-tumor necrosis factor (TNF)-a (1:100; NOVUS Biologicals, CO, USA), or rabbit anti-cleaved caspase 3 (1:100; Cell Signaling Technology Inc., Danvers, MA, USA) for $120 \mathrm{~min}$. The resultant immune complexes were then detected with a polymer reagent (Histofine Simple Stain MAX PO; Nichirei Bioscience, Tokyo, Japan). The binding of the primary antibodies to their specific antigens was visualized with the diaminobenzidine kit (Dako, Carpinteria, CA, USA) and imaged on a light microscope equipped with a Nikon Eclipse 80i camera using the Lumina Vision software (ver. 3.0, Mitani, Tokyo, Japan)

\section{Immunofluorescent staining}

After being subjected to fixation and then rinsed with PBS, the sections were pre-incubated with $10 \%$ normal

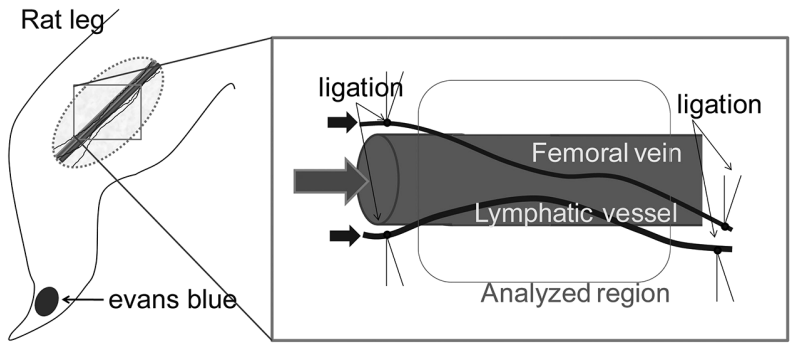

Fig. 1 Experimental animals and procedures. In brief, $0.2 \mathrm{ml}$ of Evans blue dye was subcutaneously injected into the dorsalis pedis arteries of 8-week-old male SpragueDawley rats. The lymphatic vessels around the femoral veins in the mid-thigh were identified based on the Evans blue staining. The lymphatic vessels were ligated by placing 8-0 monofilament sutures at both the proximal and distal ends of the target region, and the surrounding perivascular tissue was not surgically manipulated. The target region was used as a model of lymphatic insufficiency, whereas the equivalent tissue in the contralateral thigh was used as the control tissue.

goat serum (Nichirei Bioscience) and incubated overnight at $4{ }^{\circ} \mathrm{C}$ with mouse anti-podoplanin $(1: 100$; ReliaTech $\mathrm{GmbH}$ ) or rabbit anti-cleaved caspase 3 antibodies (1:100; Cell Signaling Technology Inc.). Immunoreactivity was visualized using Alexa Fluor 488-conjugated antimouse immunoglobulin G and Alexa Fluor 594-conjugated anti-rabbit immunoglobulin G (Invitrogen, Carlsbad, CA, USA). All Alexa Fluor-conjugated secondary antibodies were diluted 200-fold before use. The slides were stained with the appropriate secondary antibody. Cell nuclei were stained with 4-6-diamidino-2-phenylindole, and the cells were imaged using an inverted microscope (BZX-700; Keyence, Osaka, Japan).

\section{IMS}

The IMS protocol, which is briefly explained below, has been described previously. ${ }^{7}$ Eight- $\mu$ m-thick longitudinal frozen sections for the IMS were thaw-mounted onto indium tin oxide-coated glass slides (Bruker Daltonics, Bremen, Germany) and dried at room temperature. A total of $500 \mu \mathrm{L}$ of 2,5-dihydroxybenzoic acid solution was sprayed onto the sections. We performed IMS using a MALDI-time-of-flight (TOF)/TOF-type instrument (Ultraflex II TOF/TOF; Bruker Daltonics) equipped with a 355-nm Nd:YAG laser at a repetition rate of $200 \mathrm{~Hz}$. Data were acquired with a step size of $20 \mu \mathrm{m}$ in positiveion mode (reflector mode). All spectra were acquired automatically using the Flex Imaging software (Bruker Daltonics), which was also used to create ion images.

\section{Statistical analysis}

The results are summarized using descriptive statistics, and all data are expressed as the mean \pm standard deviation. 


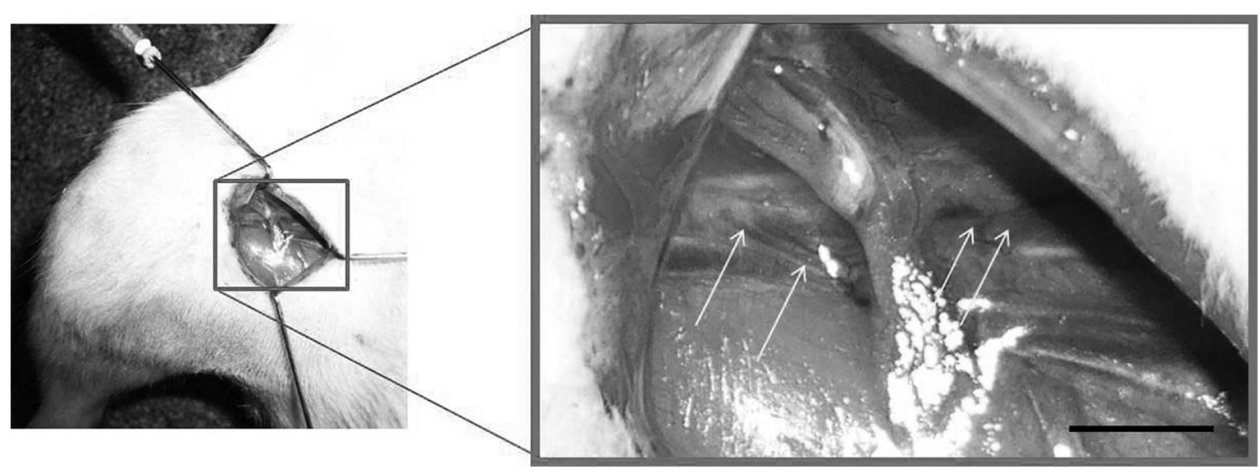

Fig. 2 Intraoperative view. The white arrows indicate lymphatic vessels. Scale bar $=1 \mathrm{~mm}$.

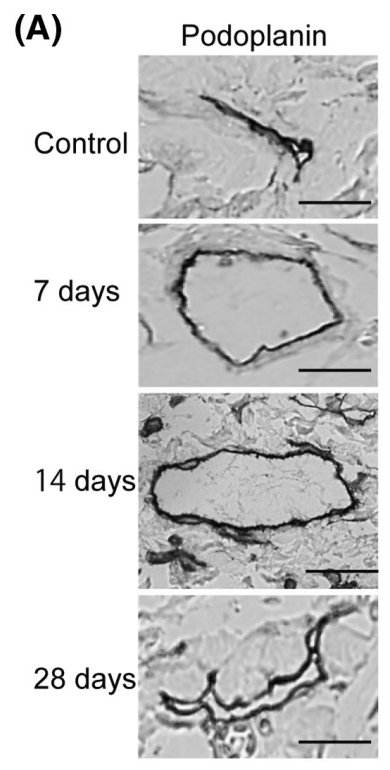

(B) $(\mu \mathrm{m})$

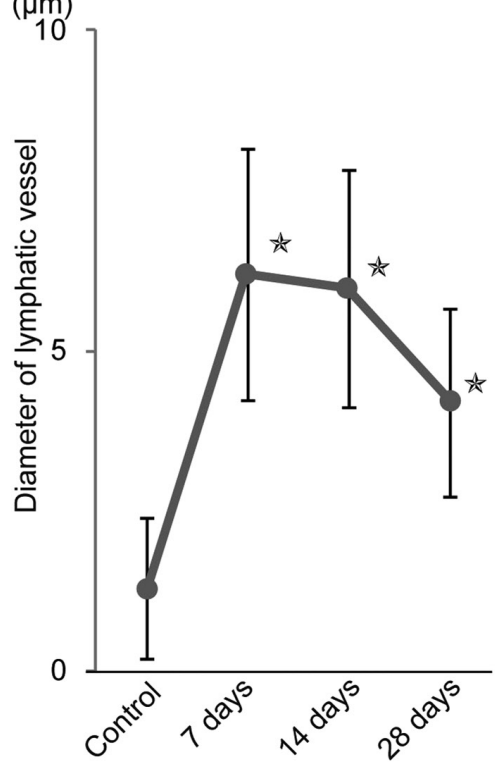

(C)

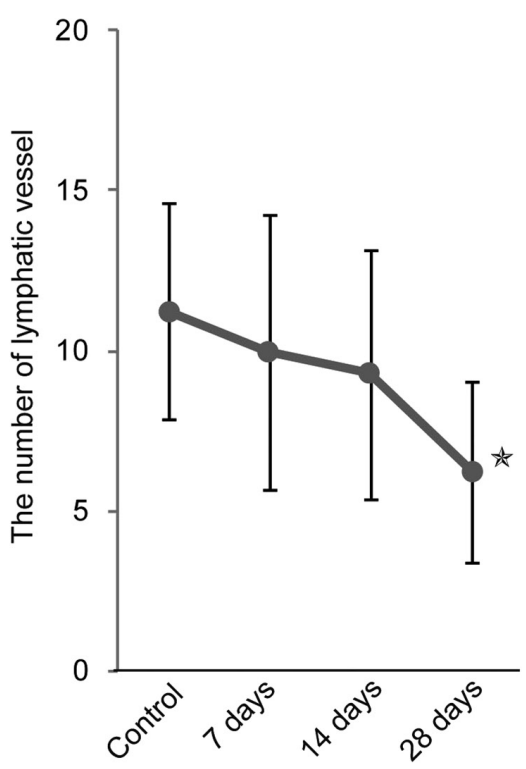

Fig. 3 Dilation and loss of lymphatic vessels in the experimental animals. (A) An immunohistological study showed that the dilated lymphatic vessels in the lymphatic insufficiency model tissue were positive for podoplanin. Scale bar = $5 \mu \mathrm{m}$. (B) At 7, 14, and 28 days after the procedure, the lymphatic vessels in the lymphatic insufficiency model tissue exhibited significantly greater diameters than those in the control tissue ( ${ }^{*} P<0.05$ ). (C) At 28 days after the procedure, the number of lymphatic vessels in the lymphatic insufficiency model tissue was lower than that seen in the control tissue $\left(n=5,{ }^{*} P<0.05\right)$. The diameters and number of lymphatic vessels differed significantly between the groups according to the Tukey-Kramer test; ${ }^{*}<0.05$ indicates a significant difference $(n=5$, each group).

The significance of inter-group differences in the diameter or number of lymphatic vessels, wall thickness, or ion intensity was determined using the Tukey-Kramer test. $P$ values of $<0.05$ were considered significant. All statistical analyses were performed using the StatView 5.0 software (SAS Institute, Tokyo, Japan).

\section{Results}

\section{Presence of lymphatic insufficiency in the animal model}

Upon exposing the femoral vein and subcutaneously injecting Evans blue dye into the dorsum of the foot, we readily identified some lymph vessels around the vein (Fig. 2). During the immunohistological study, the model tissues were positively stained for podoplanin, which is a lymphatic vessel marker (Fig. 3A), and the ligated lymphatic vessels exhibited significantly larger diameters than the control vessels after the procedure (Fig. 3B). On the other hand, the number of lymphatic vessels was lower in the model tissue than in the control tissue at 28 days (Fig. 3C). This was considered to be indicative of insufficient drainage of the lymph that had flowed from the extracellular fluid into the lymph vessels. ${ }^{8,9)}$ Normal lymph vessels are straight in form, but in cases of lymphostasis, their lumens become enlarged, as is seen in 

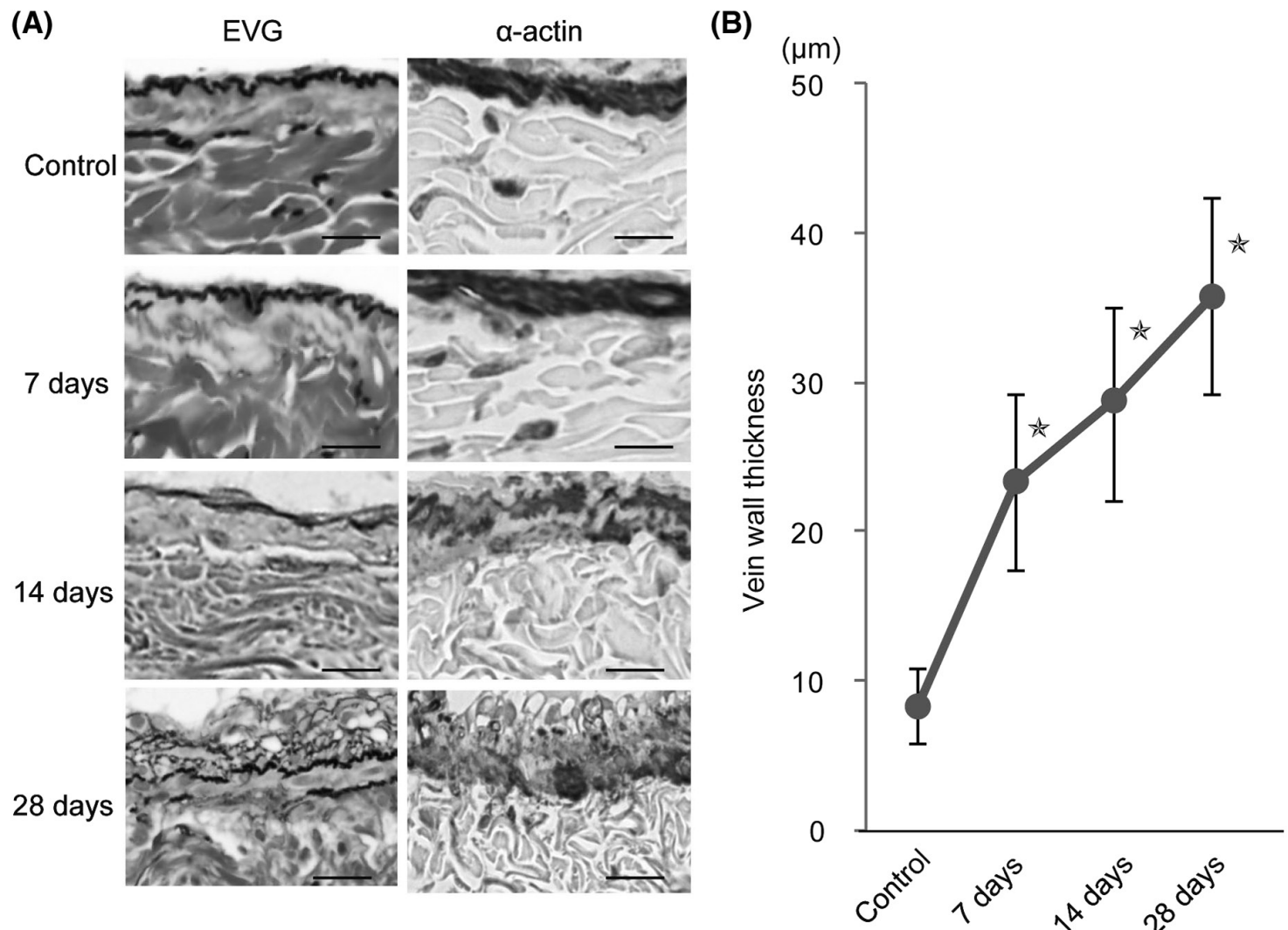

Fig. 4 Vein wall degeneration: Changes in vein wall thickness and the leakage and accumulation of lymphatic fluid. (A) and (B) Quantitative measurements of the thickness of the intima and media of the vein wall were carried out. At 7, 14, and 28 days, the intima and media of the veins in the lymphatic insufficiency model tissue were significantly thicker than those of the veins in the control tissue $\left({ }^{*} P<0.05\right)$. EVG, Elastica van Gieson; Scale bar $=20 \mu \mathrm{m}$. Significant differences in vein wall thickness were detected between the groups according to the Tukey-Kramer test; * $P<0.05$ indicates a significant difference $(n=5$, each group).

Fig. 3A. As the dilation in this model persisted for 28 days, we used it as a model of lymphostasis around the venous system.

\section{The thickness of the vein wall was affected by lymphostasis}

Next, we performed a histological analysis to identify the vein wall changes caused by lymphostasis. After the procedure, the intima and media of the model veins were thicker than those of the control veins (Fig. 4). These histopathological changes were similar to those seen in great saphenous veins affected by venous reflux in patients with VV.

\section{Characteristic distribution of lipid molecules around the vein wall}

Lymphatic vessels carry out the important role of transporting proteins and lipids. Using IMS, which is uniquely capable of lipid imaging, we found that in the model tissue lipid molecules were localized in the vein wall and perivenous tissue after lymphostasis had been induced. In addition, IMS revealed the marked accumulation of lysophosphatidylcholine (LPC) (1-acyl 16:0), phosphatidylcholine (PC) (diacyl 16:0/20:4), and triglycerides (TG) (52:2) in the walls of the model veins after the procedure (Fig. 5A). In the model tissue, inflammatory lipid molecules gradually accumulated in the vein wall and perivenous tissue (Fig. 5B). On the other hand, there were no differences in the localization of PC (diacyl 16:0/18:1) or other molecules (data not shown) between the model and control tissue.

\section{Detection of TNF- $\alpha$ around hyperplastic adipocytes in the perivascular tissue}

The prevalence of adipocytes in the model perivenous tissue increased markedly after the procedure (Figs. 6A and $6 \mathrm{~B}$ ), and the stroma surrounding these adipocytes was positive for TNF- $\alpha$ (Fig. 6C).

\section{Cleaved caspase 3 expression in lymphatic vessels}

An immunohistological study showed that cleaved caspase 3 , which is an apoptotic marker, was specifically 
(A)
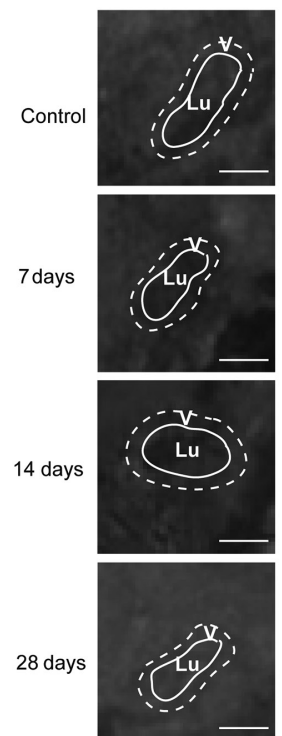
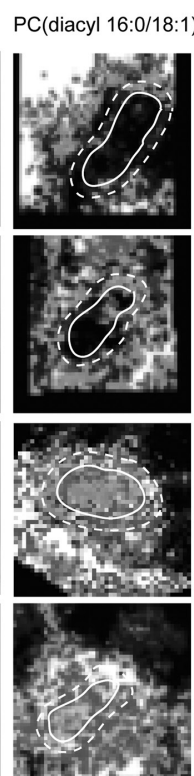
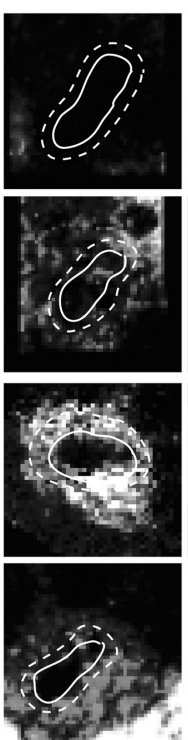

PC(diacyl 16:0/20:4)
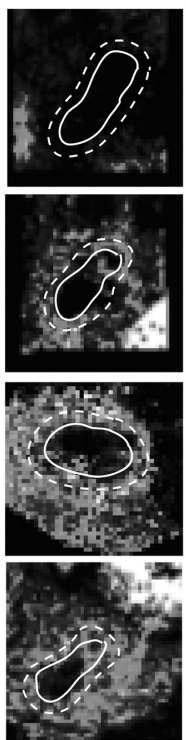

TG(52:2)

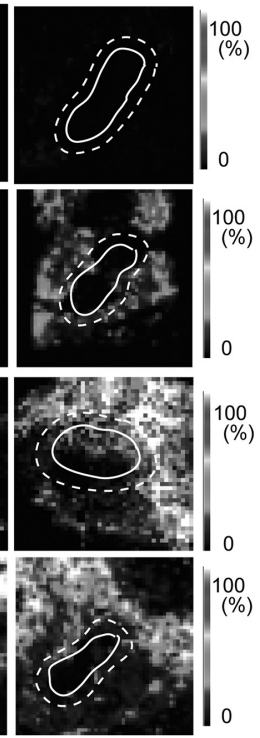

(B)

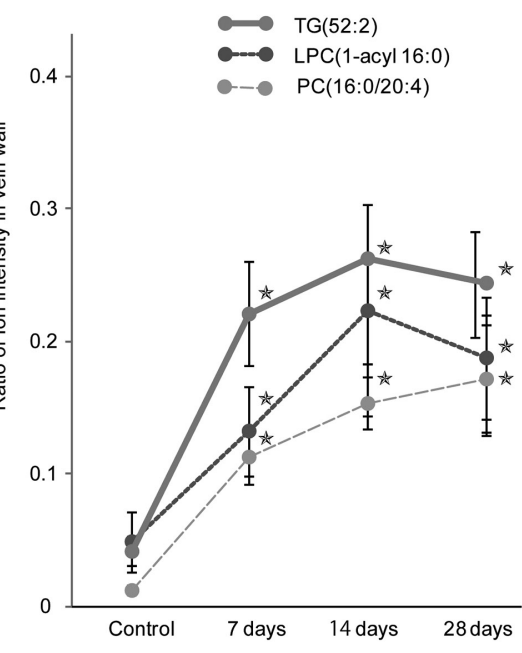

Fig. 5 Imaging mass spectrometry. (A) Distribution of LPC (1-acyl 16:0), PC (diacyl 16:0/20:4), and TG (52:2) in the vein wall. The solid line indicates the inner edge of the vein wall, and the dotted line shows the outer edge of the vein wall. The white broken line corresponds to the outer circumference of the vein wall. (B) Ion intensity of LPC (1-acyl 16:0), PC (diacyl 16:0/20:4), and TG (52:2) in the vein wall. Significant differences in ion intensity were detected between the groups according to the Tukey-Kramer test; ${ }^{*} P<0.05$ indicates a significant difference $(n=5$, each group). LPC: Iysophosphatidylcholine; Lu: Iumen; PC: phosphatidylcholine; TG: triglycerides; V: vein wall

(A)

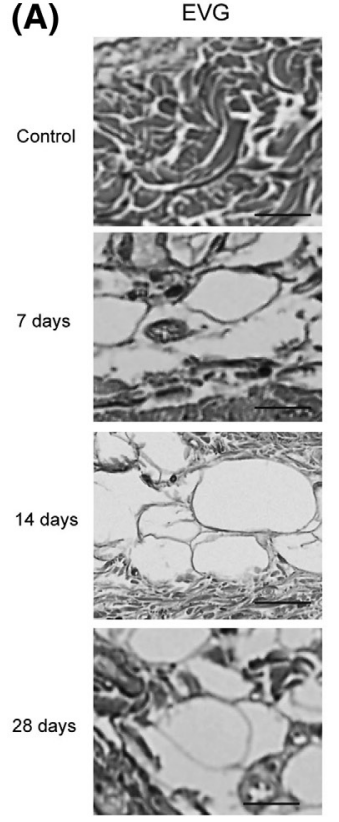

(B)

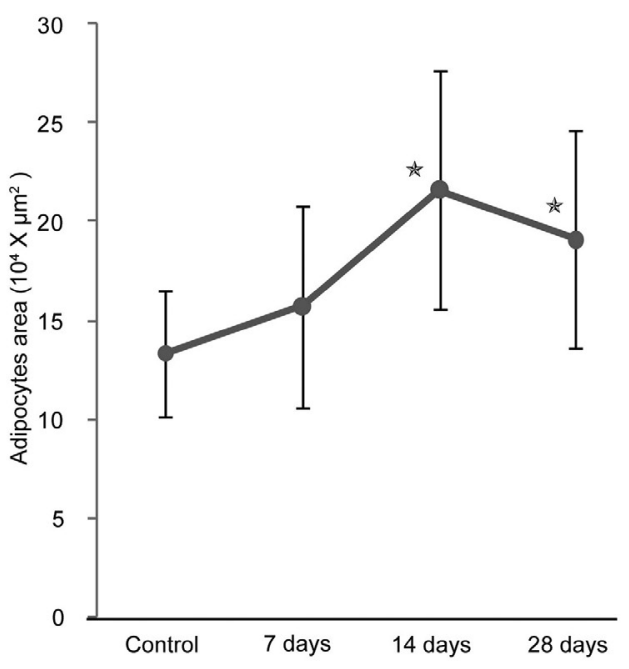

(C)
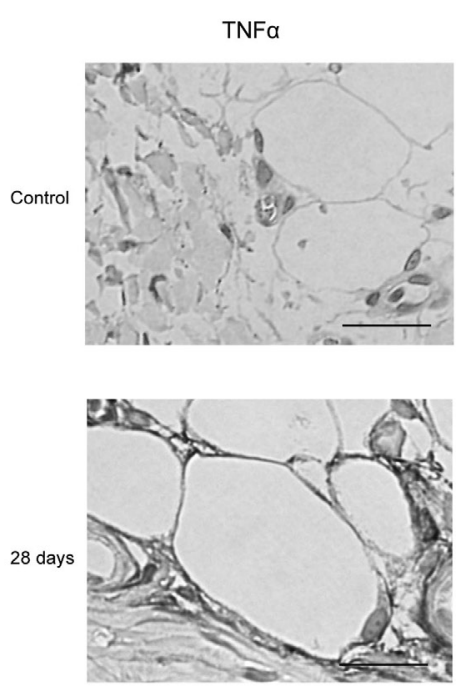

Fig. 6 TNF- $\alpha$ was detected around the hyperplasic adipocytes in the perivascular tissue. (A) The number of hyperplastic adipocytes was markedly increased in the TNF $\alpha$-positive region at 7,14 , and 28 days. EVG, Elastica van Gieson; Scale bar $=50 \mu \mathrm{m}$. (B) At 7, 14, and 28 days, the total adipocyte area in the perivascular tissue located $200 \mu \mathrm{m}$ from the vein wall was significantly greater than that in the control tissue $\left({ }^{*} P<0.05\right)$. Significant differences in adipocyte area were detected between the groups according to the Tukey-Kramer test; ${ }^{*} \mathrm{P}<0.05$ indicates a significant difference $(n=5$, each group). TNF: tumor necrosis factor 


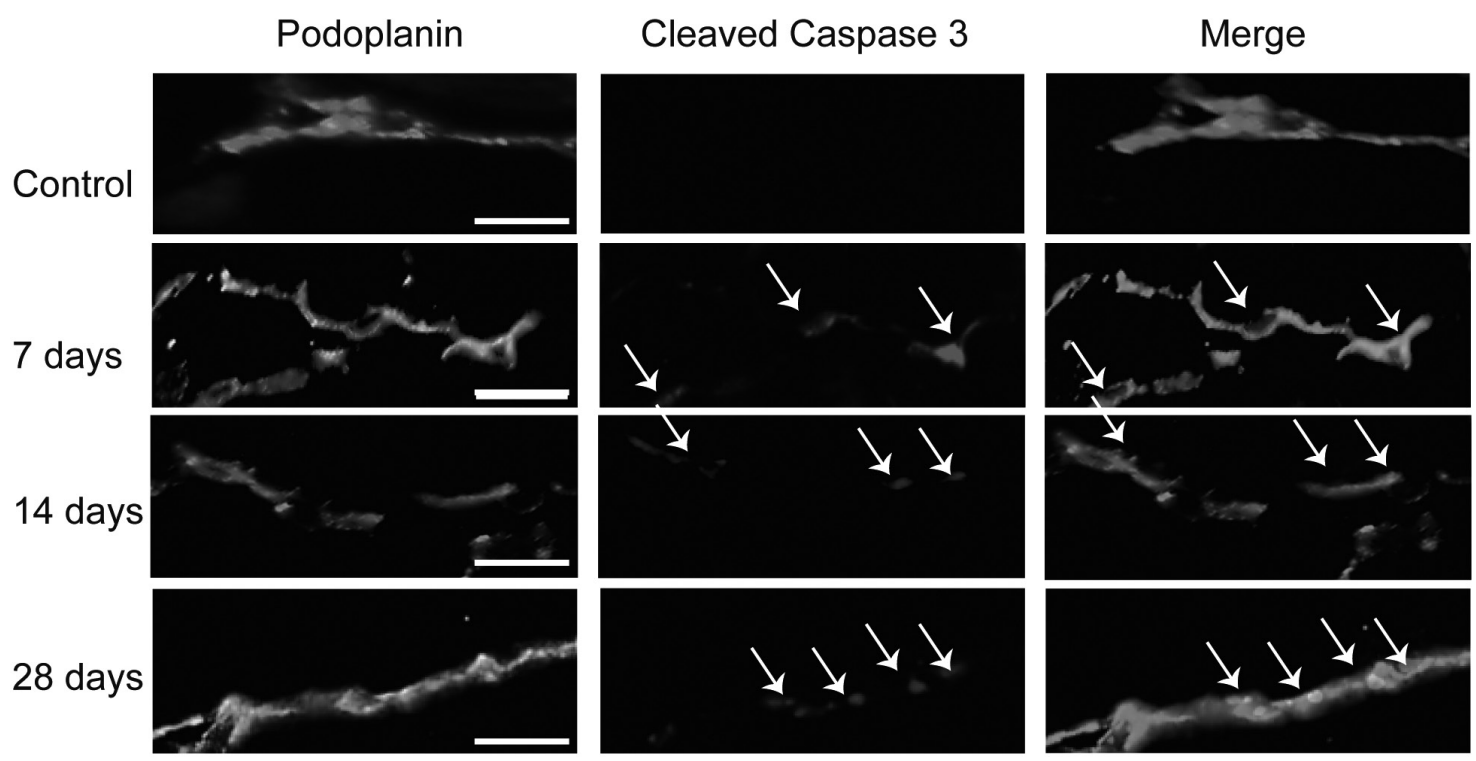

Fig. 7 Cleaved caspase 3 expression in lymphatic vessels. An immunohistological study detected cleaved caspase $3(\rightarrow)$, which is an apoptotic marker, in the dilated lymphatic vessels in the lymphatic insufficiency model tissue at 7,14 , and 28 days after the procedure. Scale bar $=5 \mu \mathrm{m}(n=5$, each group).

expressed in the dilated lymphatic vessels in the model tissue (Fig. 7). The associated apoptosis could have been responsible for the reduction in the number of lymphatic vessels seen after the procedure (Fig. 3 ).

\section{Discussion}

We created an animal model of lymphatic insufficiency and performed an experimental investigation into the physiological relationship between the veins and lymph vessels of the lower extremities. As a result, it was demonstrated that lymphostasis causes venous wall inflammation by inducing adipocyte degeneration around vein walls. Furthermore, it was suggested that sustained inflammation could lead to lymph vessel apoptosis. Lymph vessel dilation is suggestive of a rise in lymphatic pressure, which is accompanied by an increase in the intra-duct lymph flow rate. Even at 28 days after the procedure, the lymph vessel diameter of the model tissue was significantly greater than that of the control tissue. We consider that this was due to high pressure in the tissue surrounding the lymph vessels, but the detailed dynamics (i.e., lymph flow rate and tissue pressure) responsible for the observed effects remain unclear. Therefore, this issue requires further study.

Lymph vessel dysfunction causes extracellular fluid and the lipid components contained in lymph to accumulate in the surrounding tissue. ${ }^{1)}$ In the present study, IMS revealed not only the quantity, but also the distribution of lipid molecules. Specifically, it showed that in the model tissue lipid molecules accumulated in the vein wall and the surrounding tissue (Fig. 5). Also, because IMS makes it possible to perform analyses at the level of molecular species, we were able to determine that the accumulating lipid molecules comprised LPC, an inflammatory mediator, and arachidonic PC, whose side chain arachidonic acid is known to accumulate in multiple chronic inflammatory diseases. Vein wall inflammation causes medial smooth muscle cell proliferation and excess production of fibrous matter, leading to hyperplasia, which in turn reduces the elastic compliance of the vein wall. ${ }^{10,11)}$ The vein wall hyperplasia shown in Fig. 4 was considered to have been caused by the effects of lymphostasis-induced inflammation. The ex vivo animal experiments conducted in the present study indicated that the sustained passage of an excess amount of lymph through the lymphatic vessels can adversely affect the vessels' pump function and overwhelm the lymphatic system. Thus, structural deterioration of the micro-lymphatic network might contribute to venous insufficiency. ${ }^{12)}$

The accumulation of toxins, exudate, the fine particles contained in lymph, and bacteria in tissue brings about a variety of changes, including inflammation, a low oxygen environment, and fibrosis. ${ }^{13,14)}$ In this study, we demonstrated that during lymphostasis, lipid molecules accumulate in the tissue surrounding veins. Based on these findings, we will attempt to discuss the mechanism responsible for lipid molecule accumulation and metabolic abnormalities. Small adipocytes secrete specific antiinflammatory cytokines, such as adiponectin, which help to suppress inflammation. However, when excess lipids, such as fatty acids, are stored in adipocytes, hyperplasia, and phenotypically transformed adipocytes secrete 
inflammatory promoters, such as TNF- $\alpha .^{15,16)}$ Vascular wall cells are affected in a variety of ways by inflammation. For example, TNF- $\alpha$ promotes the proliferation of medial smooth muscle cells, ${ }^{17,18)}$ reduces vascular wall compliance, changes the inner lumen diameter of blood vessels, and causes abnormal blood flow. We considered that excessive accumulation of lipid molecules in lymph would cause adipocytes to degenerate and secrete proinflammatory cytokines, leading to adverse effects on the vascular wall.

The pathophysiological mechanisms responsible for VV and deep vein thrombosis involve lipid metabolism abnormalities. ${ }^{19,20)}$ Previously, we analyzed VV from patients with chronic venous insufficiency using IMS and detected the abnormal accumulation of lipid molecules in the walls of the VV, possibly due to insufficient lipid drainage by the lymphatic vessels. ${ }^{19)}$ Furthermore, we found that there are lymph vessels in the great saphenous vein wall, but the number of lymph vessels in the great saphenous vein wall was lower in the VV patients with prominent cutaneous symptoms. ${ }^{21)}$ Although the primary cause of inflammation in such tissues is not yet understood, the observed reduction in the number of lymphatic vessels could have been caused by lymphatic vessel cell apoptosis (Fig. 7). Such reductions in the number of lymphatic vessels could lead to lymphatic vessel dysfunction, and the drainage function of the surrounding tissues might subsequently deteriorate. Consequently, a vicious cycle could form in which interstitial fluid and lymph accumulate within the intracellular spaces. Vascular diseases involving chronic vascular wall inflammation, such as VV, might be related to localized lipid abnormalities. Specifically, lymph stagnation and lymph vessel dysfunction could be closely related to the inflammatory response.

It is thought that many vascular disorders are not simply diseases of the venous or lymph duct, as the functions of the venous and lymphatic systems are closely related, and hence, if the function of one declines then the other will also be affected. In this study, we sequentially observed the changes induced by artificially generated lymphostasis. In future studies, we will evaluate the effects of venous abnormalities on the adjacent lymph vessels.

\section{Conclusion}

This study indicated that the accumulation of lymphatic fluid due to insufficient lymph drainage could cause inflammation and the degeneration of the walls of adjacent veins.

\section{Disclosure Statement}

No financial assistance was received for this study. The authors have no conflicts of interest.

\section{References}

1) Semenza GL. Hypoxia pathway linked to kidney failure. Nat Med 2006; 12: 996-7.

2) Piller N. Phlebolymphoedema/chronic venous lymphatic insufficiency: an introduction to strategies for detection, differentiation and treatment. Phlebology 2009; 24: 51-5.

3) Kim DI, Huh S, Hwang JH, et al. Venous dynamics in leg lymphedema. Lymphology 1999; 32: 11-4.

4) Suzuki M, Unno N, Yamamoto N, et al. Impaired lymphatic function recovered after great saphenous vein stripping in patients with varicose vein: venodynamic and lymphodynamic results. J Vasc Surg 2009; 50: 1085-91.

5) Collins PS, Villavicencio JL, Abreu SH, et al. Abnormalities of lymphatic drainage in lower extremities: a lymphoscintigraphic study. J Vasc Surg 1989; 9: 145-52.

6) Partsch H. Investigations on the pathogenesis of venous leg ulcers. Acta Chir Scand Suppl 1988; 544: 25-9.

7) Tanaka H, Morita Y, Sugiura Y, et al. [Imaging mass spectrometry: principle and application]. Tanpakushitsu Kakusan Koso 2009; 54: 224-9.

8) Shin WS, Szuba A, Rockson SG. Animal models for the study of lymphatic insufficiency. Lymphat Res Biol 2003; 1: 159-69.

9) Galanzha EI, Tuchin VV, Zharov VP. Optical monitoring of microlymphatic disturbances during experimental lymphedema. Lymphat Res Biol 2007; 5: 11-27.

10) Elsharawy MA, Naim MM, Abdelmaguid EM, et al. Role of saphenous vein wall in the pathogenesis of primary varicose veins. Interact Cardiovasc Thorac Surg 2007; 6: 219-24.

11) Ducasse E, Giannakakis K, Speziale F, et al. Association of primary varicose veins with dysregulated vein wall apoptosis. Eur J Vasc Endovasc Surg 2008; 35: 224-9.

12) Franzeck UK, Haselbach P, Speiser D, et al. Microangiopathy of cutaneous blood and lymphatic capillaries in chronic venous insufficiency (CVI). Yale J Biol Med 1993; 66: 37-46.

13) Schander A, Downey HF, Hodge LM. Lymphatic pump manipulation mobilizes inflammatory mediators into lymphatic circulation. Exp Biol Med (Maywood) 2012; 237: 58-63.

14) Olszewski WL. Pathophysiological aspects of lymphedema of human limbs: I. Lymph protein composition. Lymphat Res Biol 2003; 1: 235-43.

15) Coppack SW. Pro-inflammatory cytokines and adipose tissue. Proc Nutr Soc 2001; 60: 349-56.

16) Bruun JM, Pedersen SB, Kristensen K, et al. Effects of pro-inflammatory cytokines and chemokines on leptin production in human adipose tissue in vitro. Mol Cell Endocrinol 2002; 190: 91-9.

17) Miao CY, Li ZY. The role of perivascular adipose tissue in vascular smooth muscle cell growth. Br J Pharmacol 2012; 165: 643-58.

18) Tanaka H, Sukhova G, Schwartz D, et al. Proliferating arterial smooth muscle cells after balloon injury express TNF-alpha but not interleukin-1 or basic fibroblast growth factor. Arterioscler Thromb Vasc Biol 1996; 16: $12-8$. 
19) Tanaka H, Zaima N, Yamamoto N, et al. Imaging mass spectrometry reveals unique lipid distribution in primary varicose veins. Eur J Vasc Endovasc Surg 2010; 40: 657-63.

20) Ageno W, Di Minno MN, Ay C, et al. Association between the metabolic syndrome, its individual components, and unprovoked venous thromboembolism: Results of a patient-level meta-analysis. Arterioscler Thromb Vasc Biol 2014; 34: 2478-85.

21) Tanaka H, Zaima N, Sasaki T, et al. Loss of lymphatic vessels and regional lipid accumulation is associated with great saphenous vein incompetence. J Vasc Surg 2012; 55: 1440-8. 